\title{
Diffusivity determination in bulk materials on nanometric length scales using neutron reflectometry
}

\author{
E. Hüger, ${ }^{1,}{ }^{*}$ J. Rahn, ${ }^{1}$ J. Stahn, ${ }^{2}$ T. Geue,${ }^{2}$ and H. Schmidt ${ }^{1,3}$ \\ ${ }^{1}$ Institute of Metallurgy, Thermochemistry and Microkinetics Group, Clausthal University of Technology, \\ Robert-Koch Str. 42, D-38678 Clausthal-Zellerfeld, Germany \\ ${ }^{2}$ Laboratory for Neutron Scattering, Paul-Scherrer-Institute, CH-5232 Villigen PSI, Switzerland \\ ${ }^{3}$ ZFM-Centre for Solid State Chemistry and New Materials, Leibniz University Hannover, Germany
}

(Received 5 March 2012; revised manuscript received 7 May 2012; published 4 June 2012)

\begin{abstract}
An approach based on neutron reflectometry and isotope heterostructures is presented in order to determine self-diffusivities in bulk materials on small length scales of 1-10 nm. The method is demonstrated for lithium self-diffusion in $\mathrm{LiNbO}_{3}$ single crystals at low temperatures of 200 and $250{ }^{\circ} \mathrm{C}$ using ${ }^{6} \mathrm{LiNbO}_{3}$ (amorphous film) / ${ }^{\text {nat }} \mathrm{LiNbO}_{3}$ (single crystal) structures for analysis. Lithium diffusivities are derived from neutron reflectivity patterns in good agreement with results obtained by secondary ion mass spectrometry on the same type of samples but on larger length scales up to $90 \mathrm{~nm}$, as given in literature. In addition, neutron reflectivity simulations were performed in order to investigate the influence of diffusion length and scattering length density on the quality of the results. The limitation of the method is discussed.
\end{abstract}

DOI: 10.1103/PhysRevB.85.214102

PACS number(s): 61.05.fj, 66.30.-h, 77.84.Bw

\section{INTRODUCTION}

Self-diffusion is a fundamental matter transport process in solids, playing a dominant role in the preparation, processing, and application of various materials. ${ }^{1}$ The determination of self-diffusivities on very short length scales of $\sim 1 \mathrm{~nm}$ is important to understand the transport properties of nanostructured $^{2}$ and metastable materials ${ }^{3,4}$ as well as of thin films, ${ }^{5,6}$ especially at low temperatures. ${ }^{7}$ Neutron reflectometry (NR) is a reliable method to measure diffusion length on the (sub)nanometer scale, as demonstrated for metallic, 2,6 intermetallic, ${ }^{10,11}$ semiconducting, ${ }^{7,9}$ and ceramic $^{3-5}$ thin films.

In order to carry out NR-based experiments, so-called isotope heterostructures of the material under investigation are used, ${ }^{2-7}$ which are deposited by sputtering or molecular beam epitaxy on appropriate substrates [see Fig. 1(a)]. In the simplest case, two layers of chemical identical materials $(A B)$, which have, however, a different enrichment of stable isotopes $\left({ }^{n} A B /{ }^{m} A B\right)$ are deposited. Due to the different bound coherent neutron scattering lengths of the two isotopes, a characteristic interference pattern is recorded in the neutron reflectivity. Annealing induces isotope interdiffusion (no chemical interdiffusion), which modifies the reflectivity. Simulations with a suitable software program package such as PARRATT32 (Ref. 8) allows one to extract self-diffusivities. Note that for the arrangement of Fig. 1(a) the mutual isotope interdiffusion results in a symmetric broadening of the isotope depth profiles. In order to increase the sensitivity of the method, self-diffusion experiments are often done with isotope multilayers, where several (typically 10-20) of the bilayers of Fig. 1(a) are combined to a stack. Here, characteristic Bragg peaks are formed in the reflectivity pattern and the decrease of this peak during annealing is analyzed. ${ }^{2-7,9-11}$ This efficient approach has the drawback that it only works for as-deposited thin films of a certain material with a thickness between 10 and $500 \mathrm{~nm}$ and not for bulk materials. Such thin films are often in a nonequilibrium state after deposition, and equilibrium properties can hardly be determined.
Here, a methodology is presented to improve the NR technique for application on equilibrium bulk materials, for instance, single crystals. The basic idea of this approach is sketched in Fig. 1(b). Chemical identical materials $(A B)$ with different isotope enrichment but also with different diffusivities will be combined. On a single crystal $\left({ }^{m} A B\right)$ with a natural isotope abundance (main isotope ${ }^{m} A$ ) a thin layer of a material ${ }^{n} A B$ enriched with the isotope ${ }^{n} A$, but with a higher diffusivity is deposited. This might be, for example, an amorphous modification of the single-crystalline material with the same chemical composition. Due to the lower mass density of the amorphous structure, faster diffusivities are expected in the deposited layer. Consequently, an asymmetric broadening of the isotope profiles is expected. Each isotope ${ }^{m} A$ that enters the amorphous region from the single crystal during annealing is instantly homogenously distributed across the whole sputter layer due to its high mobility. In contrast, the isotope ${ }^{n} A$ penetrates the single crystal from the amorphous region slowly. For short diffusion lengths, a complementary error function as isotope penetration profile is expected, as sketched in Fig. 1(b). Due to the short diffusion length in the single crystal in the order of $1-10 \mathrm{~nm}$, the constant isotope fraction $\left[{ }^{n} A\right]$ in the amorphous layer is not significantly modified if the layer thickness, $L$, is considerably larger than the diffusion length, $d$, inside the crystal. The main point is, that the modification of the reflectivity profile by diffusion is dominated by the diffusion of the ${ }^{n} A$ isotope into the single crystal. As will be shown, this allows the determination of the self-diffusivities in the single crystal, because the scattering length density in the amorphous region is only negligibly modified.

The approach will be illustrated for lithium self-diffusion experiments in lithium niobate $\left(\mathrm{LiNbO}_{3}\right)$ single crystals. $\mathrm{LiNbO}_{3}$ is an indispensable material in advanced photonics and nonlinear optics. ${ }^{12-14}$ For many applications, self-diffusion of the constituents is of high importance, especially at low temperatures. ${ }^{12}$ The formation, stability, and dissociation of defect clusters $^{15}$ that trigger physical properties such as the Curie temperature, the ferroelectric coercive field, and photorefractive properties ${ }^{12}$ are closely related to diffusion 
(a)

$\left[{ }^{\mathrm{n}} \mathrm{A}\right]$

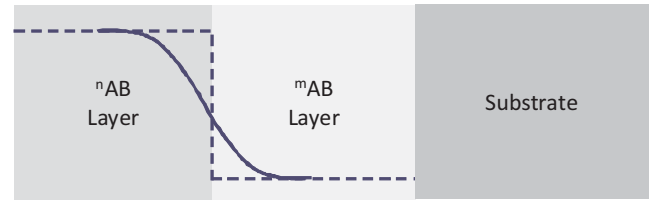

(b)

$\left[{ }^{\mathrm{n}} \mathrm{A}\right]$

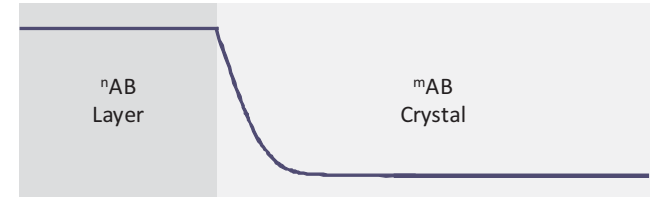

FIG. 1. (Color online) Schematic sketch of a diffusion experiment based on isotope heterostructures. $\left[{ }^{n} A\right]$ is the relative fraction of the isotope ${ }^{n} A$. (a) Homogeneous interdiffusion of the isotopes ${ }^{n} A$ and ${ }^{m} A$ between two chemical identical, but isotope-enriched layers $\left({ }^{n} A B /{ }^{m} \mathrm{AB}\right)$ deposited on a substrate (dashed line: as-deposited; full line: annealed). (b) Inhomogeneous interdiffusion of the isotopes ${ }^{n} A$ and ${ }^{m} A$ between a layer $\left({ }^{n} A B\right)$ deposited on a bulk crystal $\left({ }^{m} A B\right)$ with different diffusivities. The layer ${ }^{n} A B$ has a significantly higher diffusivity than the crystal.

properties. ${ }^{16}$ Despite a secondary ion mass spectrometry (SIMS) study, ${ }^{17}$ lithium tracer diffusion studies in $\mathrm{LiNbO}_{3}$ single crystals are absent at temperatures below $700^{\circ} \mathrm{C}$ (Ref. 18) due to the low diffusivity which is expected in that range. In the present study, we give experimental data that demonstrate that diffusivities can be determined by NR in single crystals on diffusion length scales between 1 and $8 \mathrm{~nm}$. In addition, PARRATT32-based simulations were done in order to discuss the limits of the introduced approach.

\section{EXPERIMENTAL DETAILS}

For the diffusion experiments, $\sim 50 \mathrm{~nm}$ thin isotopeenriched ${ }^{6} \mathrm{LiNbO}_{3}$ films were deposited by ion-beam sputtering on commercial $c$-axis-oriented ${ }^{\text {nat }} \mathrm{LiNbO}_{3}$ single crystals (CrysTec, Berlin) with a $\mathrm{Li}_{2} \mathrm{O}$ content of 48.6 mol \%. ${ }^{17}$ In order to unambiguously determine which effect results from the mutual interdiffusion of the ${ }^{6} \mathrm{Li}$ and ${ }^{7} \mathrm{Li}$ isotopes, a control experiment is launched, where a layer of nat $\mathrm{LiNbO}_{3}$ with a similar thickness is deposited on the single crystal.

Ion-beam sputtering was performed using a commercial setup (IBC 681, Gatan) equipped with two Penning ion sources. Deposition was done at $5 \mathrm{keV}$ and at a current of $\sim 200 \mu \mathrm{A}$ in argon at an operating pressure of $5 \times 10^{-5}$ mbar. The base vacuum was better than $5 \times 10^{-7}$ mbar. The deposition rate was $\sim 0.03 \mathrm{~nm} / \mathrm{s}$. Details on sputter target preparation are given elsewhere. ${ }^{17}$

The isothermal diffusion anneals were done in ambient air at $200{ }^{\circ} \mathrm{C}$ for $2 \mathrm{~min}, 10 \mathrm{~min}, 2 \mathrm{~h}$, and $8 \mathrm{~h}$ and at $250{ }^{\circ} \mathrm{C}$ for $4 \mathrm{~min}, 15 \mathrm{~min}$, and $32 \mathrm{~min}$. A conventional home-built resistance furnace or alternatively, a rapid thermal annealing setup (AO 500, MBE, Germany) was used for annealing. During such anneals the sputtered $\mathrm{LiNbO}_{3}$ layers stay amorphous. This was demonstrated by grazing incidence $\mathrm{x}$-ray diffractometry on thicker sputter layers on silicon substrates. ${ }^{17}$ Crystallization anneals of an amorphous sample at $500{ }^{\circ} \mathrm{C}$ showed that single-phase polycrystalline $\mathrm{LiNbO}_{3}$ is formed without any indication of impurity phases. ${ }^{17}$ This demonstrates that films and single crystals have approximately the same chemical composition.

Neutron reflectivity patterns were recorded at the timeof-flight spectrometer AMOR (SINQ, PSI Villigen). The simulations were done using the PARRATT32 code. ${ }^{8}$ For the simulations a resolution of $\triangle Q z / Q=5 \%$ was used. Recent SIMS $^{19}$ experiments found at least eight orders of magnitude higher Li self-diffusivities in amorphous $\mathrm{LiNbO}_{3}$ films than in single crystals, ${ }^{20}$ making the system perfectly suited for our purposes.

\section{RESULTS AND DISCUSSION}

\section{A. Diffusion experiments}

Figure 2 compares experimental reflectivity patterns of a bare ${ }^{\text {nat }} \mathrm{LiNbO}_{3}$ single crystal and a single crystal with a sputtered ${ }^{\text {nat }} \mathrm{LiNbO}_{3}$ layer on top in the as-deposited and annealed state together with PARRATT32 simulations. The bare ${ }^{\text {nat }} \mathrm{LiNbO}_{3}$ single crystal can be perfectly fitted by PARRATT32 using a neutron scattering length density (SLD) of $4.25 \times$ $10^{-6} \AA^{-2}$ that corresponds to a mass density of $4.6 \mathrm{~g} / \mathrm{cm}^{3}$ and a surface roughness of $0.6 \mathrm{~nm}$. For the sample with the ${ }^{\text {nat }} \mathrm{LiNbO}_{3}$ layer on top we observe the formation of characteristic fringes in the pattern, which are due to the interference of neutrons reflected at the surface and the film/ single-crystal interface, respectively. The reflection at the film/crystal interface results from the fact that the amorphous film has a lower mass density than the single crystal. The isotope concentrations are identical in crystal and film. The experimental reflectogram can be described best by PARRATT32 using a thickness of $37.5 \mathrm{~nm}$ and a SLD of $3.3 \times 10^{-6} \AA^{-2}$ (mass density: $3.6 \mathrm{~g} / \mathrm{cm}^{3}$ ) for the sputtered ${ }^{\text {nat }} \mathrm{LiNbO}_{3}$ film. The parameters of the underlying single crystal are identical to those obtained for the bare crystal. In order to obtain a

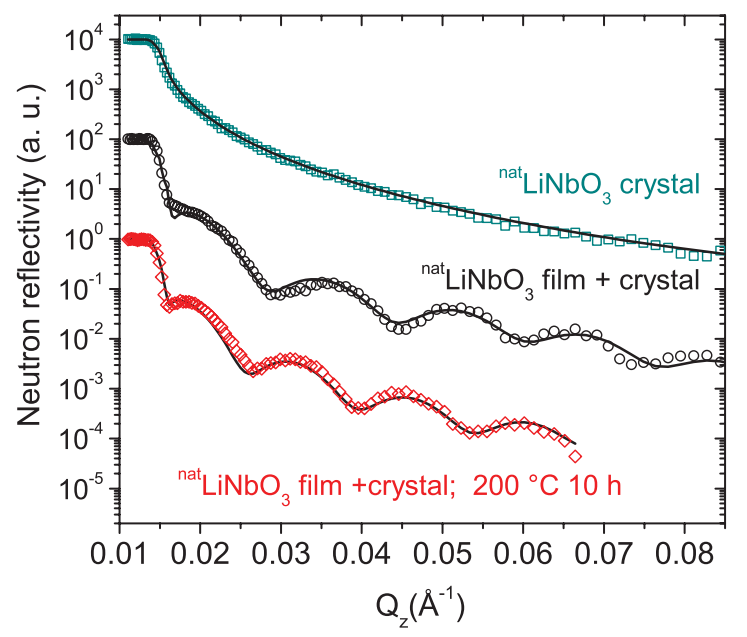

FIG. 2. (Color online) Measured NR patterns (open symbols) and corresponding PARRATT32 simulations (lines) for a bare nat $\mathrm{LiNbO}_{3}$ single crystal, for the single crystal plus deposited ${ }^{\text {nat }} \mathrm{LiNbO}_{3}$ film, and for the latter arrangement annealed at $200{ }^{\circ} \mathrm{C}$ for $10 \mathrm{~h}$. For clarity the data are shifted in intensity. For further details see text. 
TABLE I. Parameters of the PARRATT32 simulations for ${ }^{x} \mathrm{LiNbO}_{3}$ sputtered on ${ }^{\text {nat. }} \mathrm{LiNbO}_{3}$ single crystals $(x=$ nat or 6$)$ as shown in Figs. 2, 3, and 6. For the as-sputtered layers and the annealed ${ }^{\text {nat. }} \mathrm{LiNbO}_{3}$ layer, the simulations are based on a three-layer arrangement: surface layer/sputter layer/ single crystal. For the annealed ${ }^{6} \mathrm{LiNbO}_{3}$ layers, the simulations were performed using the model presented in Fig. 5. The SLD of the semi-infinite single crystal is $4.25 \times 10^{-6} \AA^{-2}$ and the corresponding surface/interface roughness is $0.6 \mathrm{~nm}$ for all samples.

\begin{tabular}{|c|c|c|c|c|c|c|c|}
\hline \multirow[b]{2}{*}{ Type } & \multicolumn{3}{|c|}{ Surface layer } & \multicolumn{3}{|c|}{ Sputter layer } & \multirow{2}{*}{$\begin{array}{c}\text { Diffusion } \\
\text { length }(\mathrm{nm})\end{array}$} \\
\hline & Thickness (nm) & $\operatorname{SLD}\left(\AA^{-2}\right)$ & Roughness (nm) & Thickness (nm) & $\operatorname{SLD}\left(\AA^{-2}\right)$ & Roughness (nm) & \\
\hline nat. $\mathrm{LiNbO}_{3}$, as deposited & 10.4 & $0.4 \times 10^{-6}$ & 0.6 & 37.5 & $3.3 \times 10^{-6}$ & 0 & \\
\hline nat. $\mathrm{LiNbO}_{3}, 10 \mathrm{~h}$ at $200^{\circ} \mathrm{C}$ & & & & 42.0 & $3.3 \times 10^{-6}$ & 0.5 & \\
\hline${ }^{6} \mathrm{LiNbO}_{3}$, as deposited & 10.9 & $1.2 \times 10^{-6}$ & 0.6 & 46.0 & $3.9 \times 10^{-6}$ & 0 & \\
\hline${ }^{6} \mathrm{LiNbO}_{3}, 2 \mathrm{~min}$ at $200{ }^{\circ} \mathrm{C}$ & & & & 50.0 & $3.9 \times 10^{-6}$ & 3.0 & 1.0 \\
\hline${ }^{6} \mathrm{LiNbO}_{3}, 10 \mathrm{~min}$ at $200{ }^{\circ} \mathrm{C}$ & & & & 51.5 & $3.9 \times 10^{-6}$ & 2.8 & 1.5 \\
\hline${ }^{6} \mathrm{LiNbO}_{3}, 2 \mathrm{~h}$ at $200{ }^{\circ} \mathrm{C}$ & & & & 53.5 & $3.9 \times 10^{-6}$ & 2.4 & 3.5 \\
\hline${ }^{6} \mathrm{LiNbO}_{3}, 8$ h at $200{ }^{\circ} \mathrm{C}$ & & & & 51.5 & $3.9 \times 10^{-6}$ & 2.3 & 6.0 \\
\hline${ }^{6} \mathrm{LiNbO}_{3}$, as deposited & 9.0 & $1.2 \times 10^{-6}$ & 1.5 & 41.0 & $3.9 \times 10^{-6}$ & 0.6 & \\
\hline${ }^{6} \mathrm{LiNbO}_{3}, 4 \mathrm{~min}$ at $250{ }^{\circ} \mathrm{C}$ & & & & 48.5 & $3.9 \times 10^{-6}$ & 2.5 & 4.0 \\
\hline${ }^{6} \mathrm{LiNbO}_{3}, 15 \mathrm{~min}$ at $250{ }^{\circ} \mathrm{C}$ & & & & 47.5 & $3.9 \times 10^{-6}$ & 1.7 & 8.0 \\
\hline${ }^{6} \mathrm{LiNbO}_{3}, 32 \mathrm{~min}$ at $250{ }^{\circ} \mathrm{C}$ & & & & 47.5 & $3.9 \times 10^{-6}$ & 1.7 & $>10$ \\
\hline
\end{tabular}

good description of the reflectivity pattern of the as-deposited sample, on top of the layer with a SLD of $3.3 \times 10^{-6} \AA^{-2}$ a second small surface layer of $\sim 10 \mathrm{~nm}$ and a SLD of $0.4 \times$ $10^{-6} \AA^{-2}$ has to be introduced. This can be explained with the existence of a low mass density layer at the surface of the sputtered layer, which is formed by reaction with the environment at room temperature. All parameters of these and the following PARRATT32 simulations are given in Table I. After annealing for $10 \mathrm{~h}$, the NR pattern is modified (Fig. 2). Now, a layer thickness of $42.0 \mathrm{~nm}$ is obtained, while the SLD is not changed. However, an additional surface layer with reduced SLD is no longer necessary for an adequate description. This effect is attributed to structural relaxation taking place in the amorphous structure during annealing. The most important result of these experiments is that the SLD of the sputtered film, and consequently the mass density are not modified during annealing in the film region adjacent to the film/crystal interface. In this context, the small 10-nm-thick surface region with reduced SLD found for the as-deposited sample is not important for our further investigations in good approximation.

Figure 3 gives a reflectivity pattern of a single crystal with a ${ }^{6} \mathrm{LiNbO}_{3}$ layer on top of it together with a PARRATT32 simulation. In contrast to the already discussed result on the ${ }^{\text {nat }} \mathrm{LiNbO}_{3}$ film, the measured neutron reflectivity of the as-deposited ${ }^{6} \mathrm{LiNbO}_{3}$ film is not much different from that of the bare single crystal. Only slightly developed fringes are observed for the nonannealed sample. This is due to the fact that the difference in SLD of the ${ }^{6} \mathrm{LiNbO}_{3}$ film and of the ${ }^{n a t} \mathrm{LiNbO}_{3}$ crystal due to different isotopes is nearly balanced by the difference in mass density between the amorphous film and single crystal. By comparison to PARRATT32 simulations we get a value of $46 \mathrm{~nm}$ for the thickness and a value of $3.9 \times 10^{-6} \AA^{-2}$ for the SLD of the ${ }^{6} \mathrm{LiNbO}_{3}$ film. The latter value corresponds to a mass density of $3.6 \mathrm{~g} / \mathrm{cm}^{3}$ in good accordance with the former experiments, proving an identical mass density of ${ }^{6} \mathrm{LiNbO}_{3}$ and ${ }^{\text {nat }} \mathrm{LiNbO}_{3}$ films. Similar to the NR results for the nat $\mathrm{LiNbO}_{3}$ film system already discussed, for the as-deposited ${ }^{6} \mathrm{LiNbO}_{3}$ film also an additional surface layer with $11 \mathrm{~nm}$ thickness and a SLD of $1.2 \times 10^{-6} \AA^{-2}$ has to be introduced in order to obtain a good description. The lack of well-developed fringes corresponds to the preferable situation of SLD contrast matching. This makes the neutron reflectivity of such samples sensitive for small changes caused by ${ }^{6} \mathrm{Li} /{ }^{7} \mathrm{Li}$ interdiffusion, if the mass densities are not modified during annealing, as demonstrated.

As further visible in Fig. 3, diffusion annealing leads to an enhancement of the fringes as a function of annealing time. This is especially visible in Fig. 3(b), where the reflectivity of the ${ }^{6} \mathrm{LiNbO}_{3}$ film $/{ }^{\text {nat }} \mathrm{LiNbO}_{3}$ crystal system (termed NR sample) is normalized to that of the pure ${ }^{\text {nat }} \mathrm{LiNbO}_{3}$ crystal (termed NR crystal) and plotted on a linear scale. The effect of the fringe enhancement observed in Fig. 3 can qualitatively be explained to be the result of the penetration of ${ }^{6} \mathrm{Li}$ into the ${ }^{\text {nat }} \mathrm{LiNbO}_{3}$ single crystal, leading to an increase in SLD of the crystal close to the isotope interface [see Fig. 4(a)] as expected from the considerations in the Introduction. In amorphous ${ }^{6} \mathrm{LiNbO}_{3}$ self-diffusion is tremendously faster than in the $\mathrm{LiNbO}_{3}$ single crystal, ${ }^{19}$ meaning every ${ }^{7} \mathrm{Li}$ ion that enters the amorphous film from the crystal quickly spreads out over the whole film thickness. In contrast, Li diffusion in the single crystal is much slower, meaning a complementary error-function concentration profile of ${ }^{6} \mathrm{Li}$ penetrates into the single crystal [see Fig. 1(b)]. This results in a strong enhancement of the SLD in the crystal close to the interface [Fig. 4(a)] and in a development and enhancement of fringes (Fig. 3). ${ }^{6} \mathrm{Li}$ has a strongly different neutron scattering length $(2.00+\mathrm{i} 0.261 \mathrm{fm})$ in comparison to ${ }^{7} \mathrm{Li}(-2.22 \mathrm{fm})$, which has the highest isotope concentration of $92.5 \%$ in ${ }^{\text {nat }} \mathrm{Li}$. Consequently, the SLD of a hypothetical ${ }^{6} \mathrm{LiNbO}_{3}$ single crystal is expected to be raised to $4.95 \times 10^{-6} \AA^{-2}$ in comparison to the value of $4.25 \times$ $10^{-6} \AA^{-2}$ of a ${ }^{\text {nat }} \mathrm{LiNbO}_{3}$ crystal.

An excellent description of the experimental reflectivity of the diffusion-annealed samples in Fig. 3(a) can be achieved using the following model. A complementary error-function (erfc) -like penetration of the ${ }^{6} \mathrm{Li}$ isotope into the single crystal 

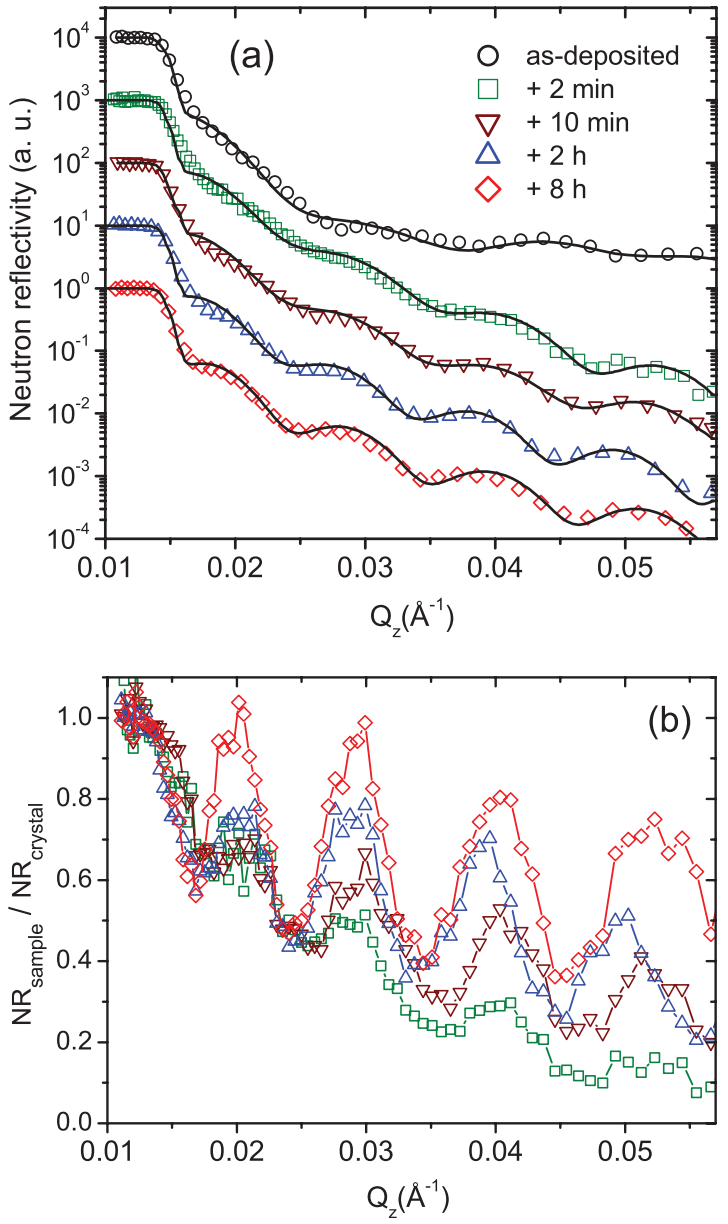

FIG. 3. (Color online) (a) Measured NR patterns (open symbols) and corresponding PARRATT32 simulations (lines) for a ${ }^{\text {nat }} \mathrm{LiNbO}_{3}$ single crystal plus deposited ${ }^{6} \mathrm{LiNbO}_{3}$ film in the as-deposited state and after annealing at $200{ }^{\circ} \mathrm{C}$ for different times. For clarity the data are shifted in intensity. (b) Normalized reflectivity patterns (NR sample/NR crystal) for a better visualization (Refs. 21-24) of the fringe enhancement during diffusion annealing. For further details refer to the text.

is assumed, according to

$$
\left[{ }^{6} \mathrm{Li}\right]=\left[{ }^{6} \mathrm{Li}_{\text {crystal }}\right]+\left(\left[{ }^{6} \mathrm{Li}_{\text {interface }}\right]-\left[{ }^{6} \mathrm{Li}_{\text {crystal }}\right]\right) \operatorname{erfc}\left(\frac{z}{d \sqrt{2}}\right),
$$

where $\left[{ }^{6} \mathrm{Li}\right]$ is the depth-dependent isotope fraction of ${ }^{6} \mathrm{Li}$, $\left[{ }^{6} \mathrm{Li}_{\text {crystal }}\right]=0.075$ is the natural concentration of ${ }^{6} \mathrm{Li}$ in the single crystal, and $\left[{ }^{6} \mathrm{Li}_{\text {interface }}\right]=0.95$ is the abundance of ${ }^{6} \mathrm{Li}$ in the deposited film and at the interface, which is assumed to be approximately constant during the diffusion experiment. Further, $d=(2 D t)^{1 / 2}$ is the diffusion length, $D$ is the diffusivity, and $t$ is the annealing time. This functional dependency is obtained from the solution of Fick's second law for diffusion across an interface from an infinite source with a constant interface concentration, ${ }^{25}$ which is a good approximation for the present situation. Consequently, the SLD, $\rho$, also shows an complementary error-function dependence, according to

$$
\rho=\rho_{\text {crystal }}+\left(\rho_{\text {interface }}-\rho_{\text {crystal }}\right) \operatorname{erfc}\left(\frac{z}{d \sqrt{2}}\right),
$$
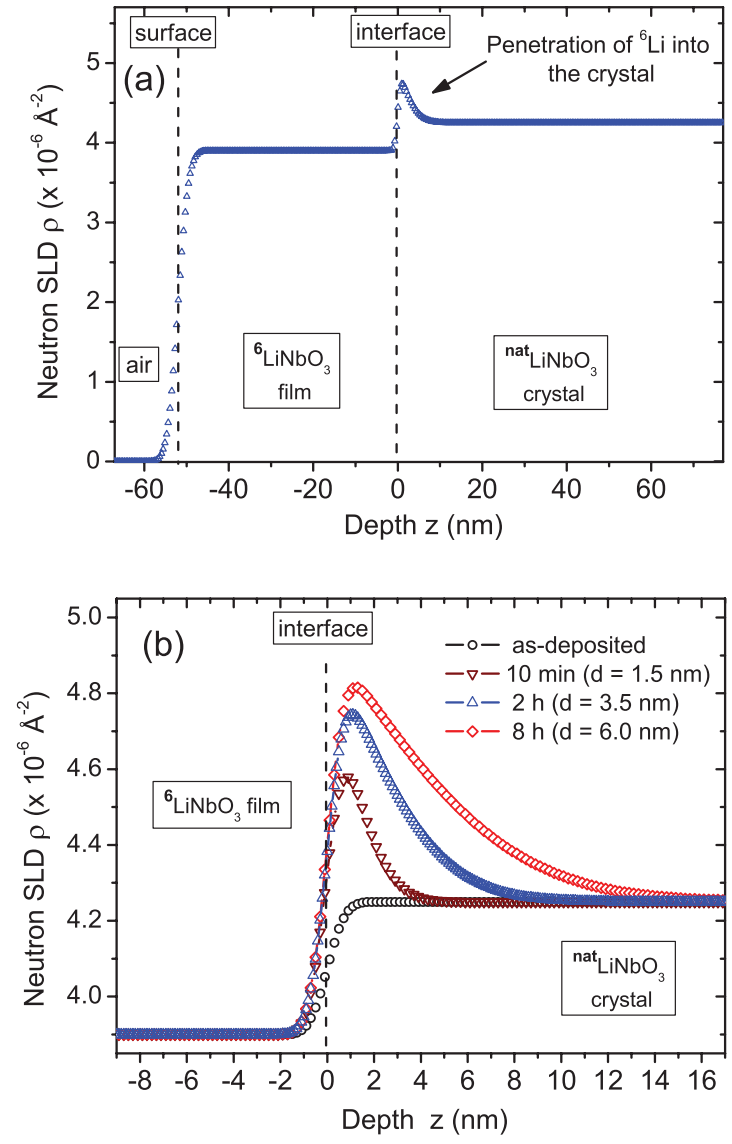

FIG. 4. (Color online) Neutron scattering length density (SLD) as obtained from the PARRATT32 simulations of Fig. 3(a). (a) Overview of the SLD for the sample annealed for $2 \mathrm{~h}(d=3.5 \mathrm{~nm})$. The space coordinate $z$ is set to zero at the film/crystal interface. (b) Enlarged view of the SLD at the film/crystal interface region for the as-deposited sample and samples annealed at $200{ }^{\circ} \mathrm{C}$ for $10 \mathrm{~min}$, $2 \mathrm{~h}$, and $8 \mathrm{~h}$. For further details refer to the text.

with $\rho_{\text {crystal }}=4.25 \times 10^{-6} \AA^{-2}$ and $\rho_{\text {interface }}=4.95 \times$ $10^{-6} \AA^{-2}$. Here, $\rho_{\text {interface }}$ is the SLD at the interface inside the crystal (not in the amorphous ${ }^{6} \mathrm{LiNbO}_{3}$ film). The incorporation of ${ }^{6} \mathrm{Li}$ into the single crystal significantly enhances the SLD as discussed. The complementary error-function dependence of the SLD according to Eq. (2) is implemented in the PARRATT32 program in the form of a bar model (see Fig. 5). The SLD was discretized by $0.7 \mathrm{~nm}$ thin layers. The length of $0.7 \mathrm{~nm}$ is approximately the distance of $\mathrm{Li}$ atoms in the $c$-axis (surface normal) direction of the $\mathrm{LiNbO}_{3}$ single crystal (termed SLD bar in Fig. 5). The surface roughness of the bare ${ }^{\text {nat }} \mathrm{LiNbO}_{3}$ single crystal has a value of $0.6 \mathrm{~nm}$. Based on this fact and to approximate more closely the analytical erfc-like decay of the SLD in the crystal, an interface roughness of $0.6 \mathrm{~nm}$ was attributed to every interface between the bars in Fig. 5. From these input parameters PARRATT32 calculates reflectivity and smooth SLD profiles (termed SLD model in Fig. 5). The dashed line corresponds to Eq. (2). As calculated in the Appendix of this work, the assumption of a constant SLD of the ${ }^{6} \mathrm{LiNbO}_{3}$ film is a good approximation for ${ }^{6} \mathrm{Li}$ diffusion lengths up to $8 \mathrm{~nm}$. 


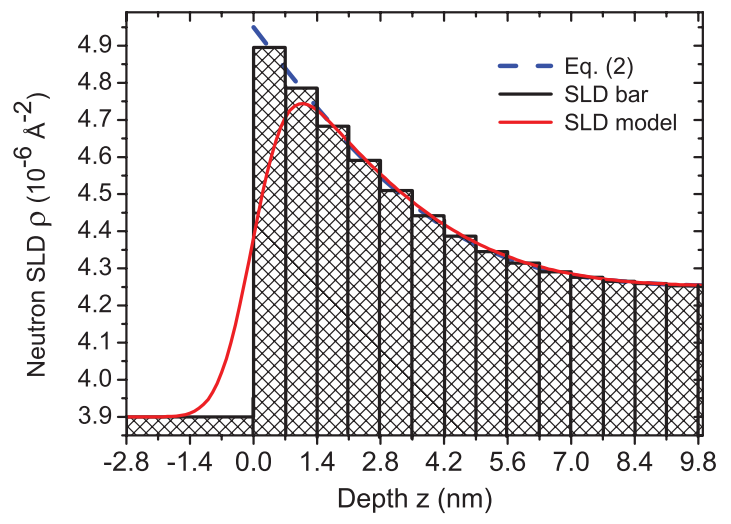

FIG. 5. (Color online) Bar model of the SLD for implementation into the program PARRATT32 for a diffusion length of $d=3.5 \mathrm{~nm}$. For further details refer to the text.

A possible problem of the present model might be that interface roughness in the Parratt formalism has some limits of validity. Generally, the interface roughness has to be smaller than the thickness of the layer. In contradiction, in the present case the interface roughness of $0.6 \mathrm{~nm}$ is close to the layer thickness of $0.7 \mathrm{~nm}$ of the discretized SLD. However, if no interface roughness is attributed to the single layers, no smooth, but an unrealistic stepwise SLD profile would be obtained. However, detailed simulations (not shown) exhibit that the calculated reflectivity is not sensitive on the interface roughness of the single layers. Assuming a roughness of 0 and $0.6 \mathrm{~nm}$, respectively, give exactly the same reflectivity, justifying the present approach.

The SLD calculated on the basis of the suggested model is illustrated in Fig. 4(b) for different diffusion lengths $d$. The corresponding calculated reflectivity is also shown Fig. 3(a). Detailed simulation parameters are given in Table I. The only quantity that changes significantly with increasing annealing time is the diffusion length (increases by a factor of 6). The height of the fringes (difference between maximum and minimum intensity) grows with increasing diffusion length.

The diffusivities are calculated directly from the diffusion length of Fig. 4(b) and Table I according to $D=d^{2} / 2 t$ and are listed in Table II. The diffusivities are identical within the error limits for different anneals. The error limits attributed to the diffusion length in Table II result from the best fit of the simulations to the experimental data. The diffusivities determined by NR on these short length scales are in agreement with values obtained by SIMS measurements done in our

TABLE II. Lithium diffusion lengths $d$ and Li self-diffusivities $D$ in $\mathrm{LiNbO}_{3}$ single crystals at $200{ }^{\circ} \mathrm{C}$ as determined by NR and SIMS (Ref. 17).

\begin{tabular}{lccc}
\hline \hline Method & Time $(\mathrm{h})$ & $d(\mathrm{~nm})$ & $D\left(10^{-21} \mathrm{~m}^{2} / \mathrm{s}\right)$ \\
\hline NR & 0.033 & $1.0 \pm 0.5$ & $4.2 \pm 4.2$ \\
NR & 0.166 & $1.5 \pm 0.5$ & $1.8 \pm 1.2$ \\
NR & 2 & $3.5 \pm 1.0$ & $0.9 \pm 0.3$ \\
NR & 8 & $6.0 \pm 2.0$ & $0.6 \pm 0.4$ \\
SIMS (Ref. 17) & 144 & 37.5 & $1.2 \pm 0.3$ \\
SIMS (Ref. 17) & 816 & 78.5 & $1.0 \pm 0.3$ \\
\hline \hline
\end{tabular}
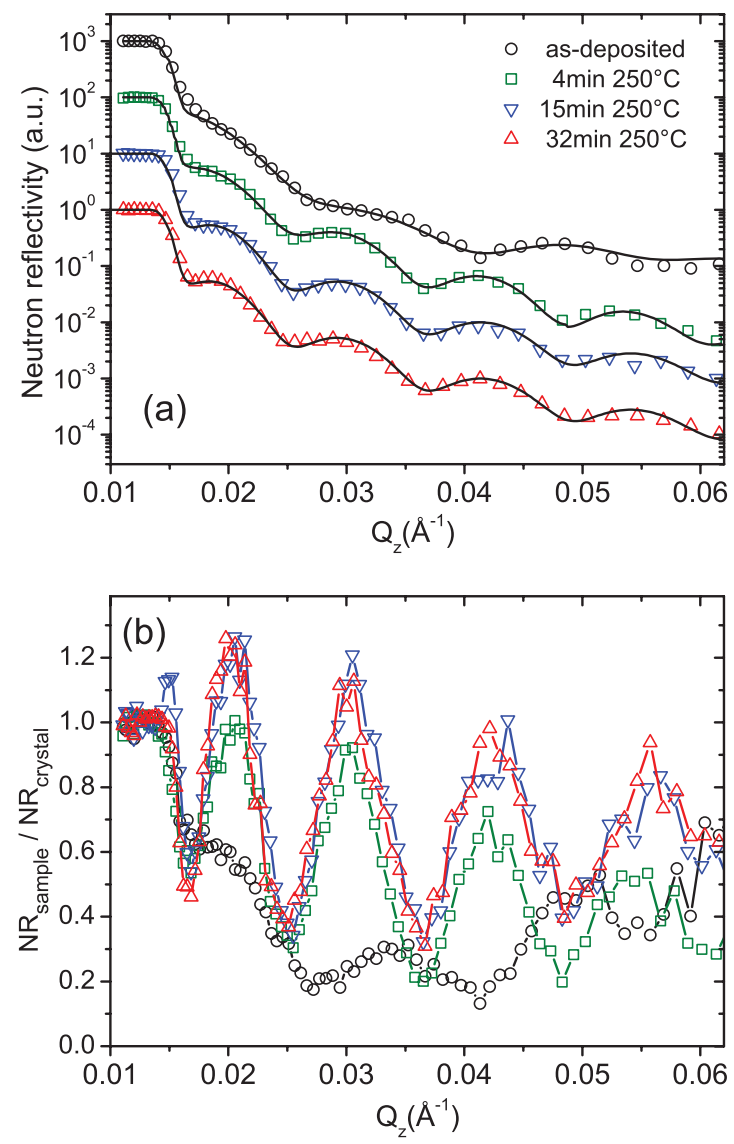

FIG. 6. (Color online) (a) Measured NR patterns (open symbols) and corresponding PARRATT32 simulations (lines) for a ${ }^{\text {nat }} \mathrm{LiNbO}_{3}$ single crystal plus deposited ${ }^{6} \mathrm{LiNbO}_{3}$ film in the as-deposited state and after annealing at $250{ }^{\circ} \mathrm{C}$ for different times. For clarity the data are shifted in intensity. (b) Normalized NR spectra (NR sample/NR crystal) for a better visualization (Refs. 21-24) of the NR fringe growth during diffusion annealing. For further details refer to the text.

laboratory ${ }^{17}$ on larger length scales of about $35-80 \mathrm{~nm}$ (Table II). These results prove that the NR approach is well suited to measure Li self-diffusivities in single crystals on small length scales. However, one has to notice that for the shortest annealing time of 2 min the obtained diffusivity has an unacceptable large error.

We further tested experimentally the presented model by performing NR measurements on ${ }^{6} \mathrm{LiNbO}_{3}$ film/ $/{ }^{\text {nat }} \mathrm{LiNbO}_{3}$ crystal samples annealed at $250{ }^{\circ} \mathrm{C}$. Figure 6 presents the measured NR patterns for diffusion anneals at $250{ }^{\circ} \mathrm{C}$ together with the corresponding PARRATT32 simulations. Similar to the experiments at $200{ }^{\circ} \mathrm{C}$, the experimental data can be successfully described by the simulations and the model given above (see Table I). There is a strong increase of the height of the fringes already after an annealing time of 4 min that corresponds to a diffusion length of $4 \mathrm{~nm}$. The NR pattern of the sample annealed for $15 \mathrm{~min}$ is due to a diffusion length of $8 \mathrm{~nm}$. During further annealing the increase in the height of the fringes saturates and a diffusion length can no longer be extracted with sufficient accuracy. This experimental finding indicates an upper limit of diffusion length determination, which will be discussed in the next section in further detail. 
TABLE III. Lithium diffusion lengths $d$ and Li self-diffusivities $D$ in $\mathrm{LiNbO}_{3}$ single crystals at $250{ }^{\circ} \mathrm{C}$ as determined by $\mathrm{NR}$ and SIMS (Ref. 17).

\begin{tabular}{lccc}
\hline \hline Method & Time $(\mathrm{h})$ & $d(\mathrm{~nm})$ & $D\left(10^{-20} \mathrm{~m}^{2} / \mathrm{s}\right)$ \\
\hline NR & 0.067 & $4.0 \pm 1.0$ & $3.3 \pm 1.7$ \\
NR & 0.25 & $8.0 \pm 3.0$ & $3.5 \pm 2.4$ \\
NR & 0.53 & & \\
SIMS (Ref. 17) & 13.8 & 56.6 & $2.8 \pm 0.8$ \\
SIMS (Ref. 17) & 62.5 & 91.2 & $1.8 \pm 0.6$ \\
\hline \hline
\end{tabular}

An overview of the results is given in Table III. Here, a good agreement between diffusivities on small length scales (NR) and on large length scales (SIMS) is also obtained.

\section{B. Additional simulations}

The aim of the present section is to find out the limits in diffusion length determination of the introduced approach. In order to achieve this, numerous NR simulations based on the PARRATT32 program package were done and analyzed. The range of diffusion lengths which are accessible by experiments will be determined. The height of the fringes developing during the diffusion experiment (see Fig. 3) may depend not only on the diffusion length, but also on the SLDs of the sputtered film $\left(\rho_{\text {film }}\right)$ of the single crystal $\left(\rho_{\text {crystal }}\right)$ and of the interface after interdiffusion, ( $\left.\rho_{\text {interface }}\right)$ [see Eq. (2)]. Simulations will be performed for a given film thickness of $52 \mathrm{~nm}$ and a surface roughness of $2 \mathrm{~nm}$. The calculations are done on the basis of the model presented in Fig. 5.

Figure 7 presents the calculated and normalized NR patterns for different diffusion lengths, ranging between 0 and $10 \mathrm{~nm}$. The simulations show that the height of the fringes is enhanced significantly by ${ }^{6} \mathrm{Li}$ diffusion from the film into the crystal as was also observed experimentally (Fig. 3). Figure 8(a) gives the integrated intensity (fringe intensity) for each fringe of Fig. 7 of dependence on the diffusion length. The numerical integration is done between two consecutive minima of a fringe. As obvious, the fringe intensity increases as a function

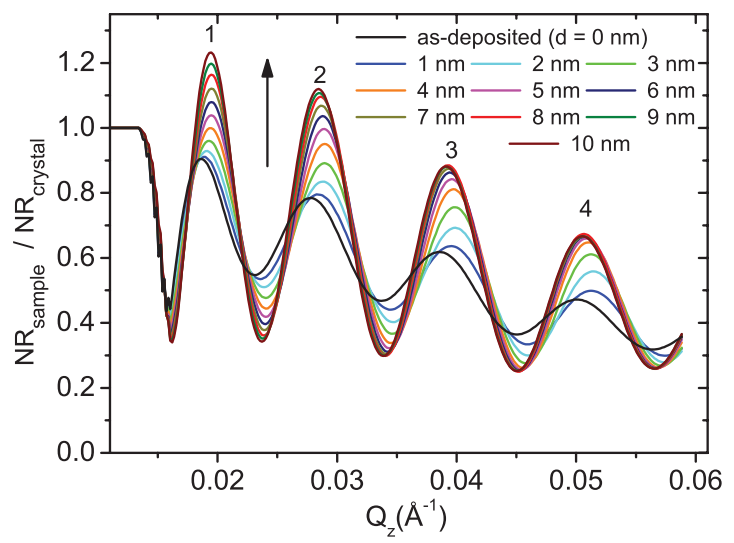

FIG. 7. (Color online) Calculated and normalized NR patterns (NR sample/NR crystal) for different ${ }^{6} \mathrm{Li}$ diffusion lengths, $d$, and for $\rho_{\text {film }}=3.90 \times 10^{-6} \AA^{-2}, \rho_{\text {interface }}=4.95 \times 10^{-6} \AA^{-2}$, and $\rho_{\text {crystal }}=$ $4.25 \times 10^{-6} \AA^{-2}$. Implementation of the bar model is done according to Fig. 5 .
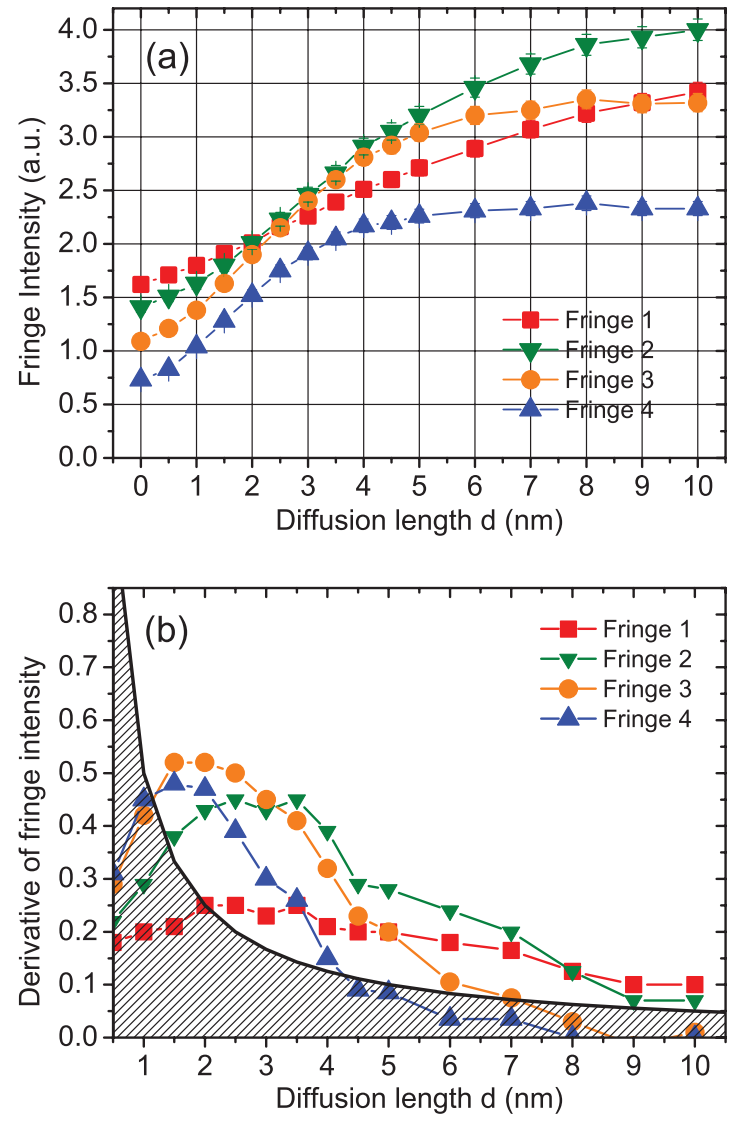

FIG. 8. (Color online) (a) Fringe intensity of the patterns presented in Fig. 7 of dependence on diffusion length. The numbers correspond to consecutive fringes for increasing $Q_{z}$ values. (b) The derivative of the curves presented in (a).

of diffusion length and becomes constant for higher values, as also observed experimentally [see Fig. 6(b)]. The starting point of this saturation depends on $Q_{z}$, and consequently on the fringe number. It occurs at higher diffusion lengths for the fringes of lower order. In the region with constant fringe intensity, the diffusivity cannot be determined by the presented approach. For a better visualization, Fig. 8(b) gives the derivative of the curves presented in Fig. 8(a). Low errors limits of the diffusion length can be achieved for regions with large slopes in Fig. 8(a), and consequently for the large derivative values in Fig. 8(b).

A constraint for a proper diffusivity determination is that the tolerable error limit of the diffusivity $D$ is less than $100 \%$. A further constraint results from the exactness of the determination of the fringe intensity $I$. Obviously, this depends on the statistical error of the reflectivity measurement. For the present case it follows from typical experimental results that the integrated fringe intensity of two measurements should differ by at least 0.25 , meaning $\Delta I>0.25$. Now, the error in diffusion lengths, $\Delta d$, can be assessed according to

$$
\Delta d=0.5 d \frac{\Delta D}{D}<0.5 d .
$$

Further we get

$$
\frac{\Delta I}{\Delta d} \approx \frac{\partial I}{\partial d}>\frac{0.5}{d}
$$




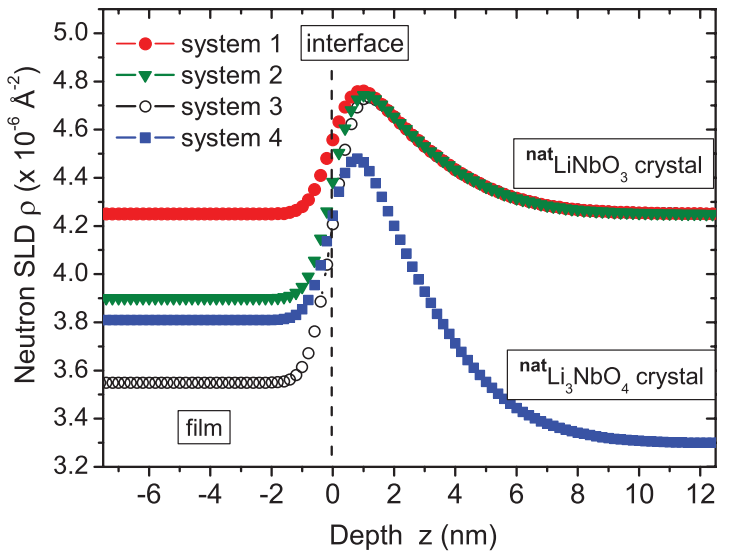

FIG. 9. (Color online) SLD as obtained from PARRATT32 simulations. Systems 1-3 correspond to $\mathrm{LiNbO}_{3}$ and system 4 corresponds to $\mathrm{Li}_{3} \mathrm{NbO}_{4}$. The presented SLDs are for a ${ }^{6} \mathrm{Li}$ diffusion length of $d=3.5 \mathrm{~nm}$.

Equation (4) is indicated in Fig. 8(b) as a shaded area. The nonshaded area represents regions where the diffusivity can be determined with an adequate error. We see that a reliable diffusivity determination is approximately possible between 1 and $10 \mathrm{~nm}$. This explains the experimental finding why it was not possible to extract a reasonable diffusivity from the NR patterns of the sample annealed for $2 \mathrm{~min}$ at $200{ }^{\circ} \mathrm{C}$, although its reflectivity pattern significantly differs from that of the as-deposited film [see Fig. 3(b)]. For the diffusion experiments done at $250{ }^{\circ} \mathrm{C}$ we had a problem to determining the diffusivity for long annealing times of $32 \mathrm{~min}$ because the corresponding diffusion length of $>10 \mathrm{~nm}$ lies within the shaded region of Fig. 8(b).

In a similar way as illustrated in the preceding section, the influence of the SLD of the sputtered film and of the crystal on the limits in diffusion length determination is elaborated for four different systems. Figure 9 illustrates the corresponding SLD profiles obtained from the PARRATT32 simulations according to the model presented in Fig. 5. The first three systems correspond to the ${ }^{6} \mathrm{LiNbO}_{3}$ film $/{ }^{\text {nat }} \mathrm{LiNbO}_{3}$ crystal system with different mass densities of the film, $\rho_{\text {film }}$, but identical values of $\rho_{\text {interface }}=4.95 \times 10^{-6} \AA^{-2}$ and $\rho_{\text {crystal }}=4.25 \times 10^{-6} \AA^{-2}$ [Eq. (2)]. In principle, different parameters during film deposition may result in ${ }^{6} \mathrm{LiNbO}_{3}$ films of a different mass density, and consequently a different value of $\rho_{\text {film }}$. The simulation results for the systems 1-3 are given in Fig. 10. As obvious, no significant differences between the three systems are found. The experimentally accessible range for diffusion length determination is $\sim 1-10 \mathrm{~nm}$. This shows that the SLD, and consequently the mass density of the deposited film is no significant parameter that may limit diffusion length determination.

Finally, as an outlook, system 4 is presented, which corresponds to another $\mathrm{Li}$ compound ${ }^{6} \mathrm{Li}_{3} \mathrm{NbO}_{4}$ film $/{ }^{\text {nat }} \mathrm{Li}_{3} \mathrm{NbO}_{4}$ crystal. $\mathrm{Li}_{3} \mathrm{NbO}_{4}$ is a compound with technological importance for, e.g., low-cost microwave dielectrics ${ }^{26}$ possessing useful luminescent characteristics. ${ }^{27} \mathrm{Li}_{3} \mathrm{NbO}_{4}$ crystallizes in the rocksalt structure, ${ }^{28}$ which is not present for $\mathrm{LiNbO}_{3}$. Here, values of $\rho_{\text {film }}=3.81 \times 10^{-6} \AA^{-2}, \rho_{\text {interface }}=4.86 \times$ $10^{-6} \AA^{-2}$, and $\rho_{\text {crystal }}=3.30 \times 10^{-6} \AA^{-2}$ are present (see also

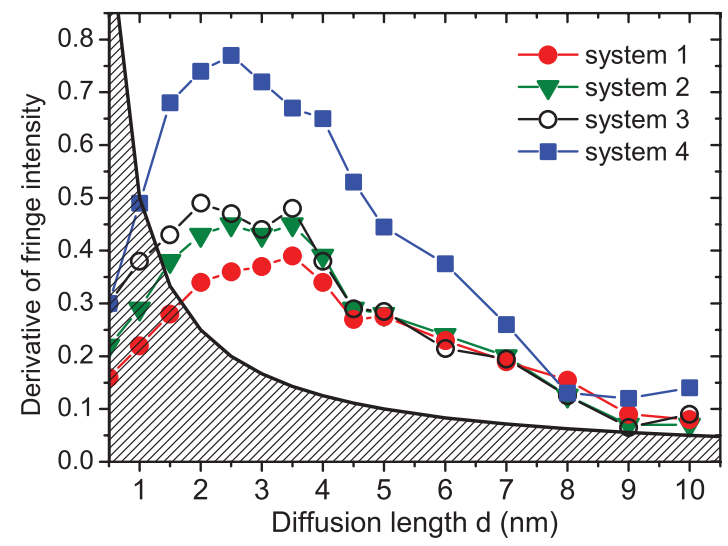

FIG. 10. (Color online) Derivative of the fringe intensity (of fringe 2) for the systems given in Fig. 9.

Fig. 9). During interdiffusion, a larger value $\rho_{\text {interface }} / \rho_{\text {crystal }}=$ 1.47 is present than for the $\mathrm{LiNbO}_{3}$ system $\left(\rho_{\text {interface }} / \rho_{\text {crystal }}=\right.$ 1.17). Figure 10 also illustrates simulation results of the $\mathrm{Li}_{3} \mathrm{NbO}_{4}$ system. Derivatives are found which are higher by a factor of 2 compared to systems 1-3. Consequently, a stronger sensitivity for diffusion determination is present than for systems 1-3. For example, a benefit of the higher derivative values of the $\mathrm{Li}_{3} \mathrm{NbO}_{4}$ system may be a reduced data acquisition time (lower necessary statistics) in order to obtain diffusivities with the same error as presented in this work for the $\mathrm{LiNbO}_{3}$ system.

\section{CONCLUSION}

We introduced an approach to determine self-diffusivities in single crystals by neutron reflectometry for small diffusion lengths of $1-10 \mathrm{~nm}$. For the experiments, a 50-nm-thick amorphous film of ${ }^{6} \mathrm{LiNbO}_{3}$ was ion-beam sputtered on ${ }^{\text {nat }} \mathrm{LiNbO}_{3}$ (0001) single crystals. The ${ }^{6} \mathrm{Li} /{ }^{\text {nat }} \mathrm{Li}$ interdiffusion leads to an enhancement of interference fringes in the NR pattern. The height of the fringes depends on the ${ }^{6} \mathrm{Li}$ diffusion length in the ${ }^{\text {nat }} \mathrm{LiNbO}_{3}$ single crystal. The Li self-diffusivity can be extracted from simulations done by the program PARRATT32. With this approach an agreement of the Li diffusivities determined by NR on small length scales, and by SIMS on large length scales was found. In addition, simulations were performed to elucidate the influence of the diffusion length and of the neutron scattering length density on diffusivity determination.

\section{ACKNOWLEDGMENTS}

This work is based on experiments performed at the Swiss spallation source SINQ, with the instrument AMOR, PaulScherrer Institute, Villigen, Switzerland. Financial support from the Deutsche Forschungsgemeinschaft (project Schm 1569/17-1) in the framework of the research unit FOR 1277 ("molife") is gratefully acknowledged. This research has also been supported by the European Commission under the 7th Framework Programme through the "Research Infrastructures" action of the "Capacities" Programme, Contract No. CP-CSA_INFRA-2008-1.1.1 No. 226507-NMI3. Thanks 
are due to B. Ruprecht and P. Heitjans (Univ. Hannover) for preparing the $\mathrm{LiNbO}_{3}$ sputter targets.

\section{APPENDIX}

In Sec. III A of the paper it was assumed for the presented model that the SLD of the ${ }^{6} \mathrm{LiNbO}_{3}$ film is constant during the diffusion anneals. This means that the influence of ${ }^{7} \mathrm{Li}$ diffusion into the ${ }^{6} \mathrm{LiNbO}_{3}$ film is neglected. In the present Appendix it will be illustrated that this assumption is justified. Since the number of ${ }^{6} \mathrm{Li}$ atoms which penetrate into the ${ }^{\text {nat }} \mathrm{LiNbO}_{3}$ crystal equals the number of ${ }^{7} \mathrm{Li}$ atoms diffusing from the crystal into the ${ }^{6} \mathrm{LiNbO}_{3}$ film, the spatially constant isotope fraction $\left[{ }^{6} \mathrm{Li}\right]$ in the ${ }^{6} \mathrm{LiNbO}_{3}$ film [see Fig. 1(b)] has to decrease as a function of time at least to a small extent. This modifies the SLD of the ${ }^{6} \mathrm{LiNbO}_{3}$ film. In the following we will calculate this modification.

In the as-deposited state the number densities of ${ }^{6} \mathrm{Li}$ ions in the film and in the crystal are given by

$$
N_{\text {film }, 0}^{6}=\left[{ }^{6} \mathrm{Li}_{\text {film }}\right]_{0} N_{\text {film }}
$$

and

$$
N_{\text {crystal }, 0}^{6}=\left[{ }^{6} \mathrm{Li}_{\text {crystal }}\right]_{0} N_{\text {crystal }},
$$

where $\left[{ }^{6} \mathrm{Li}_{\text {film }}\right]_{0}$ and $\left[{ }^{6} \mathrm{Li}_{\text {crystal }}\right]_{0}$ are the initial relative fractions of ${ }^{6} \mathrm{Li}$ and $N_{\text {film }}$ and $N_{\text {crystal }}$ are the number densities of $\mathrm{Li}$ ions in film and crystal, respectively. After ${ }^{6} \mathrm{Li}$ diffusion has started, the number of ${ }^{6} \mathrm{Li}$ ions in the film is reduced [left side of Eq. (A3)] and by the same amount that in the crystal is enhanced [right side of Eq. (A3)]. $\left[{ }^{6} \mathrm{Li}_{\text {film }}\right]$ is denoted as the spatially constant ${ }^{6} \mathrm{Li}$ fraction in the film at time $t$ after annealing. Consequently we can write

$$
\begin{aligned}
& A L_{f}\left(\left[{ }^{6} \mathrm{Li}_{\text {film }}\right]_{0}-\left[{ }^{6} \mathrm{Li}_{\text {film }}\right]\right) N_{\text {film }} \\
& \quad=\left(\left[{ }^{6} \mathrm{Li}_{\text {film }}\right]-\left[{ }^{6} \mathrm{Li}_{\text {crystal }}\right]_{0}\right) N_{\text {crystal }} A \int_{0}^{L_{c}} \operatorname{erfc}\left(\frac{z}{d \sqrt{2}}\right) d z,
\end{aligned}
$$

where $A$ is the surface area and $L_{f}$ and $L_{c}$ are the film and crystal thicknesses, respectively, and $z=0$ is located at the film/crystal interface. Now, the number densities of Li ions in film and crystal can be written as

$$
N_{\text {film }}=\frac{\rho_{\text {film }}^{m}}{M_{\mathrm{LiNbO}_{3}}^{m}} N_{A}, \quad N_{\text {crystal }}=\frac{\rho_{\text {crystal }}^{m}}{M_{\mathrm{LiNbO}_{3}}} N_{A},
$$

where $\rho_{\text {film }}^{m}$ and $\rho_{\text {crystal }}^{m}$ are the mass densities of film and crystal, respectively, $M_{\mathrm{LiNbO}_{3}}$ is the molar mass of $\mathrm{LiNbO}_{3}$ and $N_{A}$ is the Avogadro constant. Now, we obtain from Eqs. (A3)

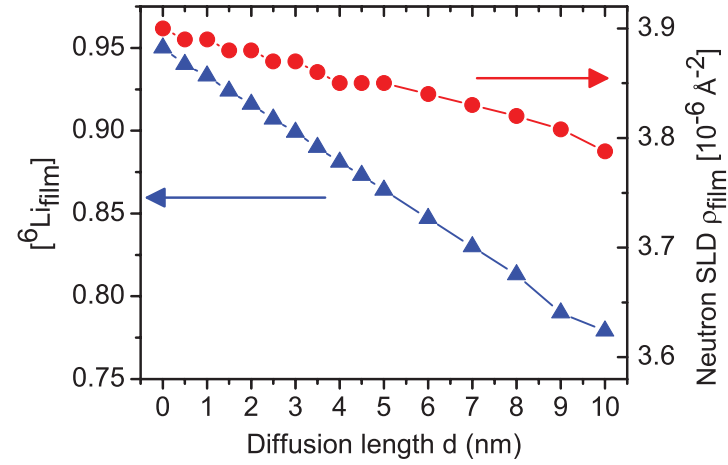

FIG. 11. (Color online) Fraction of ${ }^{6} \mathrm{Li}$ atoms, $\left[{ }^{6} \mathrm{Li}_{\text {film }}\right]$ (triangles), and SLD, $\rho_{\text {film }}$ (dots) in the ${ }^{6} \mathrm{LiNbO}_{3}$ film of dependence on the Li diffusion length, $d$. For the calculations a film thickness of $L_{f}=$ $52 \mathrm{~nm}$ was used.

and (A4),

$$
\begin{aligned}
{\left[{ }^{6} \mathrm{Li}_{\text {film }}\right]_{0}-\left[{ }^{6} \mathrm{Li}_{\text {film }}\right]=} & \left(\left[{ }^{6} \mathrm{Li}_{\text {film }}\right]-\left[{ }^{6} \mathrm{Li}_{\text {crystal }}\right]_{0}\right) \frac{\rho_{\text {crystal }}^{m}}{L_{f} \rho_{\text {film }}^{m}} \\
& \times \int_{0}^{L_{c}} \operatorname{erfc}\left(\frac{z}{d \sqrt{2}}\right) d z .
\end{aligned}
$$

For further analysis, we can use in good approximation $L_{c} \rightarrow \infty$ for the integral limit and get

$$
\int_{0}^{\infty} \operatorname{erfc}\left(\frac{z}{d \sqrt{2}}\right) d z=d \sqrt{\frac{2}{\pi}}
$$

and further,

$$
\left[{ }^{6} \mathrm{Li}_{\text {film }}\right]=\frac{\left[{ }^{6} \mathrm{Li}_{\text {film }}\right]_{0}+\left[{ }^{6} \mathrm{Li}_{\text {crystal }}\right]_{0} \sqrt{\frac{2}{\pi}} \frac{d \rho_{\text {crystal }}^{m}}{L_{f} \rho_{\text {film }}^{m}}}{1+\sqrt{\frac{2}{\pi}} \frac{d \rho_{\text {crystal }}^{m}}{L_{f} \rho_{\text {film }}^{m}}} .
$$

As obvious from Eq. (A7), the decrease of the ${ }^{6} \mathrm{Li}$ fraction in the film depends on (i) the film thickness, $L_{f}$, and (ii) the diffusion length, $d$. Using Eq. (A7), the ${ }^{6} \mathrm{Li}$ fraction in the film, $\left[{ }^{6} \mathrm{Li}_{\text {film }}\right]$ of dependence on the diffusion length, $d$, was calculated and is plotted in Fig. 11. Also shown in the plot is the SLD, which was calculated accordingly from the isotope and chemical composition with the online calculator, given in Ref. 29. For diffusion lengths up to $d=8 \mathrm{~nm}$, the decrease in SLD of the film, $\rho_{\text {film }}$, is less than $2 \%$ and can consequently be neglected. In good approximation it can be written that $\left[{ }^{6} \mathrm{Li}_{\text {film }}\right] \approx\left[{ }^{6} \mathrm{Li}_{\text {film }}\right]_{0}$.
*Corresponding author: erwin.hueger@tu-clausthal

${ }^{1}$ H. Mehrer, Diffusion in Solids, Springer Series in Solid-State Sciences, Vol. 155 (Springer, Berlin, 2007).

${ }^{2}$ H. Schmidt, S. Chakravarty, M. Jiang, E. Hüger, P. K. Parida, T. Geue, J. Stahn, U. Tietze, and D. Lott, J. Mater. Sci. 47, 4087 (2012). ${ }^{3}$ E. Hüger, H. Schmidt, T. Geue, J. Stahn, U. Tietze, D. Lott, A. Markwitz, U. Geckle, and M. Bruns, J. Appl. Phys. 109, 093522 (2011).
${ }^{4}$ E. Hüger, H. Schmidt, J. Stahn, B. Braunschweig, U. Geckle, and M. Bruns, Phys. Rev. B 80, 220101(R) (2009).

${ }^{5}$ H. Schmidt, M. Gupta, and M. Bruns, Phys. Rev. Lett. 96, 055901 (2006).

${ }^{6}$ S. Chakravarty, E. Hüger, H. Schmidt, J. Stahn, M. Horisberger, and N. P. Lalla, Scr. Mater. 61, 1117 (2009).

${ }^{7}$ E. Hüger, U. Tietze, D. Lott, H. Bracht, E. E. Haller, D. Bougeard, and H. Schmidt, Appl. Phys. Lett. 93, 162104 (2008). 
${ }^{8}$ C. Braun, PARRATT32 or the reflectometry tool, HMI, Berlin, 19971999 [http://www.helmholtz-berlin.de].

${ }^{9}$ S. M. Baker, G. S. Smith, N. J. S. Brown, M. Nastasi, and K. Hubbard, Phys. Rev. B 55, 7255 (1997).

${ }^{10}$ M. Gupta, A. Gupta, S. Chakravarty, R. Gupta, and T. Gutberlet, Phys. Rev. B 74, 104203 (2006).

${ }^{11}$ M. Rennhofer, B. Sepiol, M. Sladecek, D. Kmiec, S. Stankov, G. Vogl, M. Kozlowski, R. Kozubski, A. Vantomme, J. Meersschaut, R. Rüffer, and A. Gupta, Phys. Rev. B 74, 104301 (2006).

${ }^{12} \mathrm{~T}$. Volk and M. Wohlecke, Lithium Niobate (Springer, Berlin, 2010).

${ }^{13}$ M. Kösters, B. Sturman, P. Werheit, D. Haertle, and K. Buse, Nat. Photonics 3, 510 (2009).

${ }^{14}$ J. Shi, H. Fritze, G. Borchardt, and K.-D. Becker, Phys. Chem. Chem. Phys. 13, 6925 (2011).

${ }^{15}$ D. Birnie III, J. Mater. Sci. 28, 302 (1993).

${ }^{16}$ V. Gopalan, V. Dierolf, and D. A. Scrymgeour, Annu. Rev. Mater. Res. 37, 449 (2008).

${ }^{17}$ J. Rahn, E. Hüger, L. Dörrer, B. Ruprecht, P. Heitjans, and H. Schmidt, Phys. Chem. Chem. Phys. 14, 2427 (2012).

${ }^{18}$ H. Xu, D. Lee, S. B. Sinnott, V. Dierolf, V. Gopalan, and S. R. Phillpot, J. Phys.: Condens. Matter 22, 135002 (2010).
${ }^{19}$ D. S. McPhail, J. Mater. Sci. 41, 873 (2006).

${ }^{20}$ J. Rahn, E. Hüger, L. Dörrer, B. Ruprecht, P. Heitjans, and H. Schmidt, Z. Phys. Chem. (in press, 2012).

${ }^{21}$ T. Gutberlet and M. Lösche, in Complex Biomimetic Structures at Fluid Surfaces and Solid-Liquid Interfaces, edited by J. Fitter, T. Gutberlet, and J. Katsaras, Neutron Scattering in Biology (Springer, Berlin, 2006), p. 283.

${ }^{22}$ D. Vaknin, P. Krüger, and M. Lösche, Phys. Rev. Lett. 90, 178102 (2003).

${ }^{23}$ W. Bu and D. Vaknin, Langmuir 24, 441 (2008).

${ }^{24}$ H. Lavoie, B. Desbat, D. Vaknin, and Ch. Salesse, Biochemistry 41, 13424 (2002).

${ }^{25}$ J. Crank, The Mathematics of Diffusion (Oxford University Press, Oxford, 1975).

${ }^{26}$ J. Bian, Z. Liang, and L. Wang, J. Am. Ceram. Soc. 94, 1447 (2011).

${ }^{27}$ Y.-J. Hsiao, T.-H. Fang, S.-J. Lin, J.-M. Shieh, and L.-W. Ji, J. Lumin. 130, 1863 (2010).

${ }^{28}$ K. Ukei, H. Suzuki, T. Shishido, and T. Fukuda, Acta Crystallogr. C 50, 655 (1994).

${ }^{29} \mathrm{See}$ [http://www.ncnr.nist.gov/resources/sldcalc.html]. 